\title{
Estudo coproparasitológico da espécie Cebus libidinosus (macaco-prego)
}

\author{
[Fecal study of the species Cebus libidinosus (capuchin monkey)] \\ D.S. Alcântara ${ }^{1}$, I.L. Mendonça ${ }^{1}$, V.P. Fernandes Neto ${ }^{1}$, P.G. Carniel ${ }^{2}$, F.B. Pessoa ${ }^{3}$ \\ ${ }^{1}$ Universidade Federal do Piauí - Teresina, PI \\ ${ }^{2}$ Ibama - Porto Alegre, RS \\ ${ }^{3}$ Ibama - Teresina, PI
}

\begin{abstract}
RESUMO
Os estudos sobre parasitos gastrintestinais em primatas mantidos em cativeiros são importantes para o manejo da população de macacos e para a manutenção da saúde das pessoas que trabalham com esses animais, pois muitos desses parasitos são potentes causadores de zoonoses. Este trabalho objetivou estudar os parasitas presentes nas fezes da espécie Cebus libidinosus, criada em cativeiro. Foram utilizados 22 animais apreendidos pelo Ibama-PI, IbamA-PB e Cipama (Companhia Independente de Policiamento Ambiental do Piauí), no período de 2007 a 2009, que faziam parte do Projeto de Soltura Experimental em Ilhas Lacustres de Espécies do Gênero Cebus sp., do Ibama-PI. Foram encontrados parasitas do gênero Ancylostoma em 45\% (10/22) das amostras e do gênero Strongyloides em 23\% (05/22). No método de Willis (1921) e no método de Hoffman, o Ancylostoma sp. estava presente em $41 \%(09 / 22)$ e o Strongyloides sp. em 18\% (04/22) das amostras. A coprocultura revelou a presença de larva do gênero Ancylostoma em 50\% dos grupos estudados. A espécie Cebus libidinosus revelou-se parasitada por helmintos gastrintestinais dos gêneros Ancylostoma e Strongyloide.
\end{abstract}

Palavras-chave: primatas, macaco-prego, cativeiro, fezes, parasitas

\begin{abstract}
The studies of gastrointestinal parasites in primates kept in captivity are important for managing the population of monkeys and to maintain the health of people who work with these animals, as many of these parasites are potent causes of zoonosis. The study investigated the parasites present in the feces of Cebus libidinosus species bred in captivity. 22 animals were seized by IBAMA-PI, IBAMA-PB and CIPAMA (Independent Company of Piaui Environmental Policing) from 2007 to 2009. Hookworm parasites of the genus were found in 45\% (10/22) of samples and gender Strongiloyde in 23\% (22/05) in the Willis method (1921) and Hoffman method Ancylostoma sp. I was present in 41\% (09/22) and Strongyloides sp. 18\% (04/22) of the samples. A stool culture revealed the presence of hookworm larvae of the genus in 50\% of the groups studied. The species Cebus libidinosus proved to be parasitized by gastrointestinal helminths of Ancylostoma and Strongyloides genres.
\end{abstract}

Keywords: primates, monkey, captivity, faeces, parasites

\section{INTRODUÇ̃̃O}

Estudos sobre endoparasitas em primatas neotropicais vêm despertando interesse diante da crescente necessidade de ampliação de conhecimento da biologia das espécies ameaçadas de extinção e de planejamento de conservação delas. A presença de endoparasitas é bastante comum em animais silvestres, apresentando-se assintomática em infecções leves até manifestações clínicas graves e letalidade, principalmente em animais em cativeiro que são submetidos a um estresse alto e geralmente se encontram debilitados, interferindo na reintrodução de primatas em fragmentos florestais (Santos, 2006). Pesquisa realizada por Levacov e Jerusalinsky (2006),

Recebido em 14 de maio de 2016

Aceito em 25 de junho de 2016

E-mail: dianna1br@hotmail.com 
entre os anos de 2002 e 2006, relatou que $31,14 \%$ dos primatas existentes em Cetas (Centro de Triagem de Animais Silvestres) pertencem ao gênero Cebus, sendo este o segundo maior grupo de animais recebidos, aproximadamente 713 macacos.

O gênero Cebus pertence à ordem dos Primatas, subordem Anthropidea, infraordem Platyrrhini e família Cebidae (Rylands, 2005) e possui ampla distribuição no Brasil, ocupando ambientes tão variados quanto a Amazônia, o Cerrado, a Caatinga e a Mata Atlântica (Alberto et al., 2009).

A identificação de ovos de helmintos em fezes é feita rotineiramente na parasitologia veterinária em zoológicos de todo o mundo, sendo uma forma de diagnóstico rápido para controle das helmintoses em animais de cativeiro (Carmo e Salgado, 2003). Uma importante alternativa para o diagnóstico e estudo da helminto-fauna de animais silvestres é o processamento de fezes dos hospedeiros para obtenção dos ovos e das larvas, visando à identificação dos parasitos e a estudos relativos à sua morfobiologia (Diniz, 1997).

O objetivo do presente trabalho foi conhecer os parasitas gastrintestinais presentes nas fezes da espécie Cebus libidinosus criada em cativeiro no estado do Piauí.

\section{MATERIAL E MÉTODOS}

Foram utilizados 22 animais da espécie Cebus libidinosus, oriundos de apreensões do Ibama-PI, Ibama-PB e Cipama (Companhia Independente de Policiamento Ambiental do Piauí) e de entrega voluntária, no período de 2007 a 2009, mantidos em quarentena no Cetas do Ibama-PI, organizados em gaiolas individuais $\mathrm{e}$ identificados por meio de tatuagens na pele, que faziam parte do Projeto de Soltura Experimental em Ilhas Lacustres de Espécies do Gênero Cebus sp., do Ibama-PI. O projeto foi aprovado pelo CEEA/UFPI, sob o número 052/09.

Foram coletadas as fezes de um dia para outro, presentes em papéis esterilizados colocados embaixo das gaiolas, durante quatro dias, sempre no início da manhã, utilizando-se colheres plásticas esterilizadas e potes de vidro esterilizados para o armazenamento, lacrados e etiquetados com o número do animal correspondente à amostra e acondicionados em isopor com gelo para o transporte até o Laboratório de Sanidade Animal (Lasan) da Universidade Federal do Piauí (UFPI) para processamento das amostras. A inspeção da coleta estava incluída no Projeto de Soltura, como parte integrante do protocolo de sanidade.

As fezes coletadas foram processadas utilizandose o método de sedimentação de Hoffman (Hoffman et al., 1934) para estudo de ovos pesados, método de flutuação de Willis (1921) para estudo de ovos leves e também mediante o uso da coprocultura, com serragem de madeira esterilizada para obtenção de larvas de terceiro estágio (13). A coprocultura foi realizada em um pool de quatro grupos, cada um com cinco amostras para identificação de nematoides gastrintestinais por meio da morfologia das larvas. Em todos os métodos utilizaram-se dois gramas de fezes de cada amostra.

Foram feitas quatro lâminas de cada amostra em cada método utilizado, observadas no microscópio biocular com objetiva de 10x.

\section{RESULTADOS E DISCUSSÃO}

Todas as amostras estavam infectadas por ovos do gênero Ancylostoma e/ou do gênero Strongyloide. O gênero Ancylostoma mostrou-se predominante em todos os métodos utilizados. No método de flutuação de Willis (1921), foi possível observar a presença de ovos em uma maior quantidade de amostras, ou seja, em $45 \%$ (22/10) das amostras, e no método de Hoffman (Hoffman et al., 1934) observaram-se $41 \%$ (22/09), como mostra a Tab. 1. Os ovos do gênero Strongyloide também se apresentaram em maior quantidade de amostras no método de Willis (1921), com uma prevalência de 23\% $(22 / 05)$, enquanto no método de Hoffman (Hoffman et al., 1934) a prevalência foi de $18 \%$ das amostras (22/04).

Quanto à presença de infecção mista dos gêneros Ancylostoma e Strongyloide, esta se mostrou em maior quantidade de amostras no método de Hoffman (Hoffman et al., 1934) com 41\% (22/09), e no método de Willis (1921) com 31\% (22/07); já na coprocutlura, o gênero obtido foi o Ancylostoma, no qual em 50\% (04/02) das amostras foi identificada a presença de larva. 
Estudo coproparasitológico...

Tabela 1. Relação entre métodos coproparasitológicos e percentual de positividade de helmintos gastrintestinais em Cebus libidinus, Teresina-PI

\begin{tabular}{c|llc} 
Gêneros & Willis & Hoffman & Coprocultura \\
\hline & & & \\
Ancylostoma & $45 \%(22 / 10)$ & $41 \%(22 / 09)$ & $50 \%(4 / 2)$ \\
Strongyloides & $23 \%(22 / 05)$ & $18 \%(22 / 04)$ & 0 \\
Ancylostoma e Strongyloides & $32 \%(22 / 7)$ & $41 \%(22 / 09)$ & 0 \\
\hline
\end{tabular}

O presente trabalho demonstrou a prevalência do parasitismo causado por representantes do filo Nematoda, sendo compatível com trabalhos realizados por Santos (2006), em que foram encontrados representantes desse filo em $54,5 \%$ das 112 amostras estudadas do gênero Alouatta. Segundo Stuart et al. (1993), Ancylostoma sp. e Strongyloide sp. são os helmintos mais comuns em primatas.

Os ovos do Ancylostoma sp. possuíam formas ovoides, membrana simples e transparente , espaço claro entre a membrana e o ovo e vários blastômeros (Fig. 1A). As características dos ovos do Strongyloides sp. são de forma elipsoidal, casca delgada, extremidades polares simétricas, superfícies externas e internas lisas, camada uniforme, todos lavrados (Fig. 1B), compatíveis com as descrições feita por Diniz (1997).

A forma infectante, larva L3 ou larva filarioide, de Ancylostoma sp. tinha corpo alongado, esôfago filarioide ocupando um terço do corpo e cauda alongada (Fig. 1C), semelhante às descrições de Rey (2001).

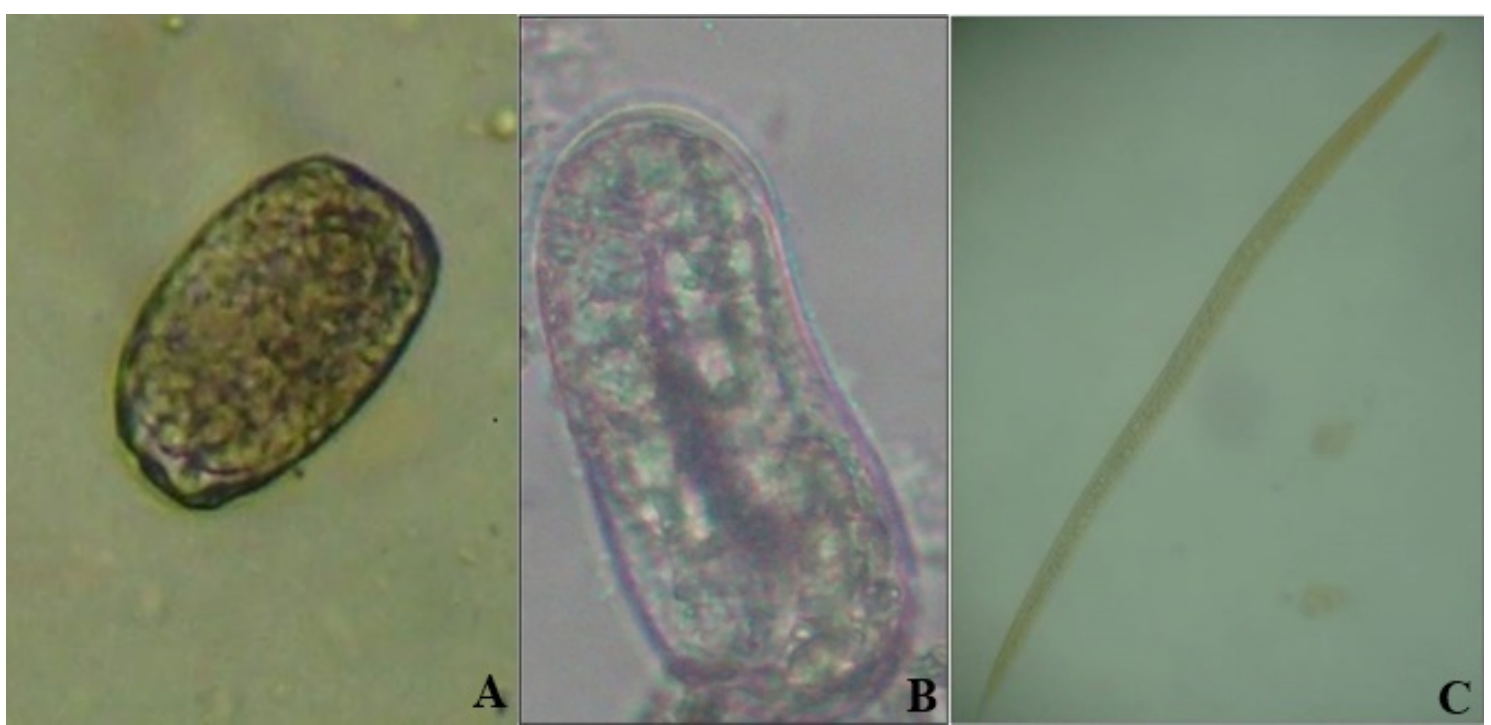

Figura 1: A- Ovo de Ancylostoma sp. B- Ovo de Strongyloides sp. C- Larva (L3) de Ancylostoma sp.

Carmo e Salgado (2003), em suas pesquisas, relataram infecção alta no gênero Callithrix por larvas Strongyloides sp., Giardia intestinais, Entamoeba coli e Entamoeba histolytica, e em saguis foi encontrado helmintos, o que não foi evidenciado na espécie Cebus libidinosus no presente trabalho. Os autores também relatam que a baixa frequência de helmintos registrada em primatas não humanos mantidos em cativeiro se deve à adoção de medidas de higiene nas gaiolas e de manejo adequado desses animais e afirmam que parasitos gastrintestinais são encontrados em animais confinados sem medidas de higiene.

Trabalhos realizados com oito primatas não humanos do gênero Alouatta por Silva et al. (2009) revelaram parasitismo por protozoários 
do gênero Giardia spp. e Cryptosporidium spp. em todos os animais pesquisados. Os autores também relatam que esses protozoários são comumente encontrados em mamíferos no Brasil. No presente trabalho, entretanto, não se observou tal ocorrência na espécie Cebus libidinosus.

Os métodos utilizados mostraram-se um meio rápido e barato para o estudo de ovos e larvas de parasitas, constituindo-se como métodos não invasivos e úteis para o estudo de parasitas em animais silvestres. Utilizou-se uma combinação de métodos com a finalidade de se obterem resultados mais precisos. A técnica de Willis (1921) mostrou-se mais eficiente para o diagnóstico do Ancylostoma sp. e Strongyloides sp. A não ocorrência de larva do gênero Strongyloide pode ser devido à baixa prevalência de ovos nas amostras.

Foram observados diferentes níveis de infecção. Para Stuart et al. (1993), diferenças na vegetação, no clima, nos hábitos dos animais e nos fragmentos florestais ou no cativeiro onde esses animais se encontravam poderiam explicar diferentes taxas de infecção. No presente estudo, não foi realizada uma estimativa precisa do grau de infecção dos animais estudados, uma vez que técnicas de quantificação de ovos não foram feitas. Santos (2006) acredita que as condições relacionadas ao ambiente podem favorecer a prevalência de infecções parasitárias, havendo prevalência maior em ambientes úmidos.

\section{CONCLUSÃO}

A espécie Cebus libidinosus revelou-se parasitada por helmintos gastrintestinais dos gêneros Ancylostoma e Strongyloides, evidenciando a necessidade da adoção de protocolos anuais de vermifugação e controle de endoparasitas, bem como manejo sanitário dos animais que vivem em cativeiro.

\section{AGRADECIMENTOS}

Os autores agradecem ao Ibama-PI e ao Ibama$\mathrm{PB}$, por concederem os animais para o presente trabalho e oferecerem apoio durante todo o processo de realização dele.

\section{REFERÊNCIAS}

ALBERTO, R.S.; IZAR, P.; KAWATA, C.M. Levantamento de primatas do gênero cebus em cativeiro no estado de São Paulo: propostas para destinação de excedentes e elaboração de guia de identificação. In: CONGRESSO DE ECOLOGIA DO BRASIL, 9., 2009, São Lourenço. Anais... São Lourenço, MG: SEB, 2009. p.17.

CARMO, A.M.; SALGADO, C.A. Ocorrência de parasitos intestinais em Callithrix sp. (Mammalia, Primates, Callithrichidae). Rev. Bras. Zoociênc., v.5, p.267-262, 2003.

DINIZ, L.S.M. Primatas em cativeiro: manejo e problemas veterinários, enfoque para espécies neotropicais. São Paulo: Ícone, 1997. p.95-104.

HOFFMAN, W.A.; PONS, J.A.; JANER, J.L. The sedimentation-concentration method in schistosomiasis mansoni. Puerto Rico J. Public Health, v.9, p.281-98, 1934.

LEVACOV, D.; JERUSALINSKY, L. Primatas brasileiros em centros de triagem: análise dos registros de 2002 a 2005. In: CONGRESSO INTERNACIONAL SOBRE MANEJO DE FAUNA SILVESTRE NA AMAZÔNIA E AMÉRICA LATINA, 7., 2006, Ilhéus. Anais... Ilhéus: UESC, 2006. CD-ROM.

REY, L. Parasitologia: parasitos e doenças parasitárias do homem nas Américas e na África, 3.ed. Rio de Janeiro: Guanabara-Koogan, 2001. 856p.

RYLANDS, A.B.; KIERULFF, M.C.M.; MITTERMEIER, R.A. Notes on the taxonomy and distributions of the tufted capuchin monkeys (cebus, cebidae) of south America. Lundiana, v.6, Suppl., p.97-110, 2005.

SANTOS, M.V.S. Levantamento de helmintos intestinais em bugio-ruivo, Alouatta guariba (primates, atelidae) na mata Ribeirão Cachoeira, no distrito de Souzas. 2006. [84f.]. Dissertação (Mestrado em Biologia Animal) - Instituto de Biologia, Universidade Estadual de São Paulo, Campinas, SP.

SILVA, A.S.; GRESSLER, L.T.; LARA, V.M. et al. Protozoários gastrintestinais em Buggios (Alouatta sp.) mantidos em cativeiro. Ciênc. Anim. Bras., v.10, p.669-672, 2009.

STUART, M.D.; STRIER, K.B.; PIERBERG, S.M.A. Coprological survey of parasites of wild muriquis, Brachyteles arachnoids, and brown Howling monkeys, Alouatta fusca. J. Helminthol. Soc. Wash., v.60, p.111-115, 1993.

WILLIS, H.H. A simple levitation method for the detection of hookworm ova. Med. J. Aust., v.11, p.375-6, 1921 . 